\title{
Felix Hausdorff: Gesammelte Werke. Band VI. Geometrie, Raum und Zahl, hg. von M. Epple (Berlin: Springer, 2021). ISBN 978-3-540-77838-7. XX 540 Seiten. 89,99€
}

\author{
Klaus Volkert
}

Angenommen: 29. März 2021 / Online publiziert: 20. April 2021

(C) Der/die Autor(en) 2021

Im Jahr 2020 kam so manches Großprojekt zu einem Ende, darunter auch eines zur Mathematikgeschichte: Es erschien der letzte Band der „Gesammelten Werke“ von Felix Hausdorff. Dieser Band, der sechste der insgesamt zehn Bände (Band I besteht aus zwei Teilbänden) umfassenden Ausgabe, ist gewidmet „Geometrie, Raum und Zeit" und wurde von Moritz Epple (Johann Wolfgang von Goethe Universität Frankfurt) herausgegeben. Er umfasst XX plus 540 Seiten und ist in gewohnt anspruchsvoller Ausstattung beim Springer-Verlag erschienen. Der Band besteht aus drei Hauptteilen: einem Essay des Herausgebers „Felix Hausdorffs Erkenntniskritik von Zeit und Raum“ (pp. 6-207), publizierte Texte aus Hausdorffs Feder (pp. 211-344) sowie unpublizierte Texte aus dem Nachlass (pp. 347-533). Ein Verzeichnis der Schriften Hausdorffs sowie ein Personenregister runden den Band ab.

Unter den publizierten Texten findet sich die einzige gedruckte Arbeit Hausdorffs zur Geometrie, nämlich die „Analytischen Beiträge zur nichteuklidischen Geometrie“ [1]. Hinzukommen zehn Besprechungen, darunter eine mit deutlichem Bezug zur Geometrie - nämlich die von Studys Geometrie der Dynamen. Weiterhin findet sich die Grabrede Hausdorffs für seinen Kollegen Study. Die unpublizierten Texte umfassen eine Ausarbeitung für die Vorlesung über Raum und Zeit (WS 1903/04), ein Manuskript über das Relativitätsprinzip und den Entwurf für einen Artikel über nichteuklidische Geometrie. Hinzu kommen noch thematisch geordnete Notizen.

Hausdorffs Name wird selten bis nie mit der Geometrie in Verbindung gebracht. Das ist auch nicht erstaunlich, denn er hat nur, wie bereits bemerkt, eine einschlä-

K. Volkert ( ()

Universität Wuppertal, Wuppertal, Deutschland

E-Mail: klaus.volkert@math.uni-wuppertal.de 
gige Arbeit hierzu veröffentlicht. ${ }^{1}$ Nach Arbeiten zur rechnenden Astronomie, zur Optik und zur Wahrscheinlichkeitsrechnung wandte sich Hausdorff Ende der 1890er Jahre vorübergehend der Geometrie zu. Dabei fand er in Leipzig mehrere Mitstreiter vor - zum einen natürlich S. Lie, den allerdings menschlich schwierigen Inhaber des Lehrstuhls für Geometrie, der nach Möbius' Tod eingerichtet worden war und mit Felix Klein besetzt wurde, aber auch den etatmäßigen, d.h. bezahlten, Extraordinarius Friedrich Engel und den etwas jüngeren Heinrich Liebmann, der sich 1899 in Leipzig habilitierte. In diesem Jahr kam O. Hölder als Nachfolger von Lie nach Leipzig, wo er am 22. Juli seine Antrittsvorlesung hielt über „Anschauung und Denken in der Geometrie“. Darin setzte sich Hölder u. a. mit der Axiomatik der Geometrie auseinander; schon einen Monat nach Hilberts Festschrift diskutierte er dessen Grundlegung der Proportionenlehre. Die Anmerkungen, die Hölder seinem Vortrag anlässlich von dessen Drucklegung (1900) beigab, zeigen eine detaillierte Kenntnis von Hilberts Axiomatik. ${ }^{2}$ Hölder könnte also Hilberts „Festschrift“ in Leipzig bekannt gemacht haben, ein Ereignis, das für Hausdorff in seinem konsequenten Formalismus wichtig war. ${ }^{3} 1904$ kam noch Karl Rohn nach Leipzig - insgesamt sicher eine anregende und aktive Umgebung, gerade was die Geometrie anbelangte. Zudem wurde die Geschichte der Geometrie, insbesondere die der nichteuklidischen, in Leipzig aktiv bearbeitet von Fr. Engel und von H. Liebmann. Gerade bei H. Liebmann gibt es Bezüge zu Hausdorff, wie schon der Titel von Liebmanns Leipziger Antrittsvorlesung „Notwendigkeit und Freiheit in der Mathematik“ [3] vermuten lässt.

Der das Buch einleitende Essay zeichnet Hausdorffs Weg zu seinem „besonnenen Empirismus“" ${ }^{*}$, wie er selbst seine Philosophie der Geometrie nannte, nach, beginnend mit der klassischen Debatte über relativen versus absoluten Raum, also bei Leibniz und Newton (bzw. Clark als seinem Stellvertreter) über Kant hin zu den Diskussionen des 19. Jhs. Ein neues Element, das in jenem Jh. ins Spiel kam, ist die (Sinnes)Physiologie, die mit H. Helmholtz einen prominenten Vertreter fand, der sich auch zu Grundlagenfragen der Geometrie äußerte. Er stellte die am Verhalten fester Körper orientierte freie Beweglichkeit in den Mittelpunkt seiner axiomatischen Bemühungen, ein Ansatz, der von S. Lie später verbessert wurde. Erkenntnistheoretisch wichtig war Helmholtz' Versuch, die Verhältnisse einer nichteuklidischen Welt

\footnotetext{
$1 \mathrm{Zu}$ Leben und Werk von Hausdorff vgl. man den Band IB der Gesammelten Werke von Hausdorff, in dem sich die sehr ausführliche (über 1000 Seiten) von E. Brieskorn und W. Purkert ausgearbeitete Biographie Hausdorffs findet. Weniger fleißige Leser wird es vielleicht freuen, dass demnächst eine von W. Purkert besorgte Kurzfassung dieses Werkes in deutscher und englischer Sprache erscheinen soll.

2 Übrigens war es der Leipziger Fr. Engel, der die „Festschrift“ für das Jahrbuch über die Fortschritte der Mathematik besprach. Engel hatte 1890 seine Antrittsvorlesung als Extraordinarius in Leipzig gehalten über ,Der Geschmack in der neueren Mathematik“. Vorlesungen und Vorträge dieser Art gab es zahlreiche, eine kleine Übersicht bietet Lorey [4, S. 6-7]. Eine genauere Untersuchung dieses Themas scheint lohnend.

3 Spuren der Auseinandersetzung mit Hilberts Axiomatik findet man u. a. in den unpublizierten Notizen an vielen Stellen (vgl. etwa p. 483 ff, p. 502) sowie in einem Brief, den Hausdorff im Oktober 1900 an Hilbert schrieb (pp. 126-128).

4 Vgl. p. 153 für genauere Ausführungen zu diesem Begriff. Ähnlich wie schon M. Pasch (1882) versucht Hausdorff Erfahrung und formale Analyse im Rahmen der Geometrie zu vereinbaren. Paschs Formulierung von der Geometrie als der exaktesten Naturwissenschaft findet sich mehrfach auch bei Hausdorff (z. B. p. 284). Seitenzahlen ohne weitere Angaben beziehen sich stets auf den hier zu besprechenden Band.
} 
anschaulich zu machen; für Hausdorff allerdings nicht mehr als ein psychologisches Faktum. Ein interessanter Hinweis des Essays ist jener auf den Petersburger Biologen Karl Ernst von Baer und seine Ideen zur „Naturalisierung Kantischer Ideen“ (p. 31). Von hier aus ergibt sich eine Beziehung zu Experimenten zum Zeitempfinden des Freiburger Psychologen Hugo Münsterberg, an denen Hausdorff als Student teilnahm (p. 34-35).

Weiter geht die Einleitung auf den Neukantianismus mit seinem Versuch ein, die neueren geometrischen Entwicklungen, insbesondere die Etablierung der nichteuklidischen Geometrie ab etwa 1860, mit der Lehre Kants, die Sätze der Geometrie seien synthetisch a priori, zu vereinbaren. Dabei kommt ausführlich Otto Liebmann zur Sprache mit seinem Hauptwerk „Die Analysis der Wirklichkeit“ (1876), dessen Lösung vereinfacht gesprochen darauf hinauslief, die nichteuklidische Geometrie als logisch möglich aber als anschaulich unmöglich einzustufen. Liebmann gilt mit seiner Schrift „Kant und die Epigonen“ $(1865)^{5}$ als einer der Begründer des Neukantianismus, ein interessanter Link zu Hausdorff ist Liebmanns bereits genannter Sohn Heinrich, der lange Jahre zusammen mit Hausdorff in Leipzig tätig war und ein versierter Geometer zudem.

Ein breites Panorama philosophischer Einflüsse wird im einleitenden Essay entworfen, wichtige Denker für Hausdorff/Mongré waren bekanntlich Fr. Nietzsche und A. Schopenhauer. Die Auseinandersetzung mit Kants Apriorismus einerseits und seiner Idee eines transzendenten Raumes (und einer transzendenten Zeit) andererseits ist ein zentraler Punkt bei Hausdorff; auch neuere Ansätze wie der logische Empirismus, insbesondere M. Schlick, werden berücksichtigt. Während der Mathematiker Hausdorff ein strenger Formalist war, dem Präzision über Alles ging, prägte Paul Mongré Begriffe wie ,transcendenter Nihilismus“ und „Chaos in kosmischer Auslese“: ein bemerkenswerter Gegensatz.

Breiten Raum im Essay nimmt eine Debatte ein, die im französischsprachigen Raum geführt wurde, von der in Deutschland aber kaum Kenntnis genommen wurde, wohl aber von Hausdorff. Hier sind Namen zu nennen wie Calinon, Delboeuf, Lechalas, Renouvier und Paul Tannery. ${ }^{6}$ Sie bildet den Hintergrund für Poincarés Artikel zur Philosophie der Mathematik, die auch im deutschsprachigen Raum eine Zeitlang viel Beachtung fanden. Was die Geometrie anbelangt, so liegen Poincarés Konventionalismus und Hausdorffs „,besonnener Empirismus“ eigentlich nicht sehr weit auseinander - die These ist in beiden Fällen, dass die Erfahrung für die Geometrie eine Rolle spiele, dass sie aber nicht die Wahl einer Geometrie erzwinge - letzteres wäre wohl die Ansicht des unbesonnenen Empiristen. Weniger gut zu Hausdorffs Auffassungen dürfte allerdings Poincarés dezidierter Kantianismus bzgl. der Arithmetik gepasst haben. Heinrich Liebmann verwies gegen Poincarés Konventionalismus, der seiner Ansicht nach besagte, ,daß die geometrischen Axio-

\footnotetext{
5 Nicht „Zurück zu Kant“, wie p. 46 behauptet wird. Der letzte geradezu programmatische Satz des Buches heißt: „Es muss auf Kant zurückgegangen werden.“ Liebmanns Position wird ausführlich in Volkert [6, S. 210-221] dargestellt.

6 Einige dieser Namen kommen auch in den unpublizierten Notizen im dritten Teil des Bandes wieder vor. Wenn auf Seite 80 des zu besprechenden Werkes in Note 257 eine Untersuchung der französischsprachigen Diskussion als ,lohnend und hilfreich“ gewünscht wird, so bleibt festzuhalten, dass diese seit vielen Jahren in Voelke [5, S. 277-319] bereits vorliegt.
} 
me praktische Festsetzungen sind, willkürliche Stempel, dem Bewußtseinsinhalt, soweit er sich auf die Außenwelt bezieht, aufgeprägt, nur von dem etwas schattenhaften Schema der Gruppe beherrscht", auf das bereits oben genannte Buch seines Vaters, „wo die Vorstellung des euklidischen Raumes als zwingend betrachtet und diese Auffassung näher analysiert wird. "7 Ob er hier auch an Hausdorff und seine Präferenz für Spielräume gedacht hat, muss mangels Belegen offen bleiben. ${ }^{8}$

Eine interessante Frage, die in der Einleitung diskutiert wird ${ }^{9}$, ist Hausdorffs Rezeption der Relativitätstheorie und deren Auswirkungen auf seine Philosophie der Geometrie, insbesondere auf seine These, dass die euklidische Geometrie die Geometrie des uns umgebenden Raumes sei. Nach Einstein war der Raum neu zu denken $^{10}$ - ein Thema, das gut zwanzig Jahre nach Hausdorff W. Threlfall in seiner Antrittsvorlesung in Dresden wieder aufgreifen sollte. ${ }^{11}$ Letztere zeigt, dass man, wollte man über den Raum nach 1905 sprechen, um Einstein nicht mehr herumkam. Das hat natürlich auch Hausdorff gesehen.

Will man wesentliche mathematische Einflüsse aufzeigen, die auf Hausdorff gewirkt haben, so kann man zwei Punkte hervorheben: die Begegnung mit Hilberts Axiomatik der Geometrie, vorgelegt in dessen Beitrag zur Festschrift anlässlich der Enthüllung des Gauß-Weber-Denkmals in Göttingen (17. Juni 1899), und die Rezeption der Mengenlehre Georg Cantors. Auf erstere kommen wir weiter unten zu sprechen, letzterer wird an vielen Stellen der Einleitung deutlich. Während Hilbert weder die Mengensprache noch die Operationen der Mengenlehre in seinen „Grundlagen“ verwandte, begann Hausdorff sogleich diese in der Geometrie zu gebrauchen (vgl. p. 130). Die Auseinandersetzung Hausdorffs mit Hilberts Grundlagen wird in der Einleitung ausführlich behandelt im Abschn. 4.1, ${ }^{12}$ man erkennt hier deutlich Hausdorffs formal-analytischen Fähigkeiten. Hilberts Festschrift hat neben Hausdorff eine größere Anzahl von Versuchen initiiert, dessen System zu verbessern oder zu verändern; dies war offensichtlich ein vielversprechendes Forschungsprogramm. Außer H. Liebmann und G. Hessenberg, die beide genannt werden, könnte man auf die im anglosächsischen Raum entstandene Schule der „,postulational analysis“ (Hausdorff selbst spricht treffend von „Axiomenforschung“ (p. 326)) hin-

\footnotetext{
7 Liebmann [3, S. 238].

8 Schlecht kommt übrigens Schopenhauer bei Liebmann weg: „Dazu kommt noch, daß der Mathematiker (z.B. Euklid) in seinem eigenen Gebiet (nach Schopenhauers Ansicht) alles Leben vernichtet, wenn er die intuitive Erkenntnis durch überflüssige Beweise stört und damit in gewisser Hinsicht gegen seine Wissenschaft noch einmal das Verbrechen begeht, was er sich schon lange gegen die Naturlehre zu Schulden kommen läßt.“ (Liebmann [3, S. 232]). Diese Position will Liebmann wohlgemerkt widerlegen.

9 Vgl. Abschn. 5 pp. 163-181.

10 Vielleicht aber auch nicht - wie Oskar Becker, ab 1931 Kollege im Fach Philosophie von Hausdorff in Bonn (nach dem Krieg vorübergehend amtsenthoben wegen seiner Schriften im Dritten Reich) in seiner Kontroverse mit H. Reichenbach und in einigen seiner Veröffentlichungen auf dem Hintergrund der Phänomenologie behauptete. Becker hat 1914 in Leipzig bei Hölder promoviert.

11 Vgl. http://www2.math.uni-wuppertal.de/ volkert/Raumproblem.pdf (aufgerufen 25.03.2021).

12 Er stammt von E. Scholz (Wuppertal). Hausdorff hatte zusätzlich zur Festschrift noch ein Exemplar der Vorlesungsausarbeitung „Elemente der Euklidischen Geometrie“ (Göttingen, WS 1898/99) von D. Hilbert zur Verfügung.

13 Vgl. Hilbert ([2] Kap. 5).
} 
weisen, im deutschsprachigen Raum auch auf Arthur Rosenthal. ${ }^{13}$ Wie Hausdorffs Publikationsverzeichnis (pp. IX-XV) zeigt, wandte er sich ab 1906 in seinen Arbeiten überwiegend der Mengenlehre und mengentheoretischen Fragen in Topologie/ Maßtheorie und Analysis zu.

Der Text von Hausdorff, der in Epples Essay mit Abstand am meisten zitiert wird, ist dessen bereits erwähnte unter dem Pseudonym Paul Mongré erschienene Schrift „Das Chaos in kosmischer Auslese“ (1898), die im Band VII der HausdorffWerke zu finden ist. Die sonstigen im sechsten Band versammelten Texte - darunter vor allem die Antrittsrede Hausdorffs - werden in den Abschn. 4.2-4.4 des Essays vorgestellt. Es seien hier ergänzende Bemerkungen zu einigen dieser Texte und zu ihren mathematikhistorischen Kontexten und Inhalten angefügt. ${ }^{14}$

Die einzige Arbeit zur Geometrie von Hausdorff, die im Druck erschien, trug den Titel „Analytische Beiträge zur nichteuklidischen Geometrie“, sie wurde am 6. März 1899 von Hausdorffs Doktorvater und Förderer H. Bruns der Sächsischen Akademie vorgelegt. ${ }^{15}$ Es handelt sich dabei in der Hauptsache um eine Darstellung der Kreisgeometrie im Rahmen der hyperbolischen Geometrie; Hausdorff gibt eine gut lesbare, fast lehrbuchartige Aufbereitung, keine neuen Ergebnisse. Das betont der Verfasser selbst in seiner Einleitung (p. 213), in der er auf eine mögliche Verwendung seiner Ausführungen in einer Vorlesung verweist. Hausdorff bearbeitet sein Thema analytisch mit Hilfe der Koordinaten von Weierstrass. ${ }^{16}$ In der ebenen hyperbolischen Geometrie sind drei Arten von Kreisen zu unterscheiden (gewöhnliche Kreise, Grenzkreise und Abstandlinien), ein Thema, das Hausdorff in seinem unveröffentlichten Artikel über nichteuklidische Geometrie genauer erläutert (vgl. pp. 364-365). Obwohl technisch in ihrem Charakter enthält die Abhandlung von 1899 doch einige interessante Hinweise allgemeinerer Natur. So heißt es im Kontext des absoluten Längenmaßes (p. 239) ${ }^{17}$ :

Man wird also gut daran thun, in jener Existenz einer absoluten Länge nichts anderes $\mathrm{zu}$ sehen als die mathematische Thatsache, dass die parabolische [d.i. die euklidische; K. V.] Maassbestimmung eine undualistische Ausartung der allgemeinen projectiven Maassbestimmung ist [...]

Damit will Hausdorff alle philosophischen Spekulationen zu dieser Frage zurückweisen, die hierin ,eine Art erkenntnistheoretischen Gegengrundes gegen die objective Möglichkeit der nichteuklidischen Geometrie gefunden“" (p. 239) zu haben meinten (Legendre war seiner Meinung nach ein Beispiel hierfür, vielleicht auch Gauß). Hier wird ein Grundthema von Hausdorff deutlich: die Verteidigung der Mathematik, genauer: ihrer Freiheit, gegen Übergriffe seitens der Philosophie;

\footnotetext{
${ }^{14}$ Das Thema „Zeit“ wird dabei ausgeklammert. Als Nietzsche-Leser war es für Hausdorff natürlich eine Herausforderung, dessen ewige Wiederkehr des Gleichen mit mathematischen Mitteln zu fassen - wie übrigens auch für O. Becker. Ein mathematisches Modell hierfür zu finden, scheint eine große Verlockung gewesen zu sein.

15 Vgl. pp. 213-266 des hier zu besprechenden Bandes.

16 Oft auch Beltrami-Weierstrass-Koordinaten genannt. Hausdorff verweist auf W. Killing, der diese Koordinaten bekannt gemacht hat - Weierstrass trug sie nur in seinem Seminar (1872) vor.

17 Vgl. auch p. 492, wo das Thema absolutes Längenmaß in Notizen von Hausdorff wieder aufgegriffen wird.
} 
vgl. hierzu auch seine Besprechung von W. Ostwalds „,Vorlesungen über Naturphilosophie“ (pp. 273-278) sowie seine Antrittsvorlesung über das Raumproblem (pp. 279-303).

An der genannten Stelle der Arbeit von 1899 kommt noch ein anderes Vorzugsthema von Hausdorff zur Sprache: das Gedankenexperiment (vgl. auch die Einleitung p. 11, 69 und 106) des vergrößerten oder verkleinerten Raumes, das u.a. aufzeigt, „die Längenmessung bleibt in dem Sinne relativ, dass eine gleichmässige Vergrösserung oder Verkleinerung aller Raumdimensionen von Seiten der Bewohner eines nichteuklidischen Raumes durch interne Messungen nicht constatirt werden kann.“ (p. 239). Dieses wohlbekannte Argument ist eine Anwendung des „Transformationsprinzips“, dem im einleitenden Essay eine wichtige Rolle zuerkannt wird (pp. 95-97). In Hausdorffs eigenen Worten (p. 97):

Jeder veränderten Anordnung entspricht nun das, was man neuerdings eine Transformation oder Abbildung des einen Raumes auf den anderen nennt. ${ }^{18}$

Letztlich geht es darum, dass die damals neue Mathematik, insbesondere Cantors Mengenlehre, alternative Denkmöglichkeiten, Spielräume, eröffnete, welche es erlaubten, die Strukturen von Zeit und Raum, etwa deren Stetigkeit ${ }^{19}$, neu zu denken; andererseits könnten Hausdorffs Bemühungen in dieser Richtung auch ein wichtiges Motiv für seine Beschäftigung z.B. mit Ordnungsstrukturen u.ä. gewesen sein, zu denen er ab 1906 publizierte. Im dritten Teil des vorliegenden Bandes finden sich unveröffentlichte Aufzeichnungen Hausdorffs, die er unter der Überschrift „Transformationsprincip“ einordnete (pp. 505-519).

Die Mathematik insgesamt ist für Hausdorff ein „logisches Experiment“ (p. 352), ihre Suche nach Voraussetzungen, die Beweisanalyse, wird verglichen mit den Analysen der Chemie. Hausdorffs Arbeit zur nichteuklidischen Geometrie scheint im Übrigen kaum Beachtung gefunden zu haben; eine Ausnahme bildete H. Liebmann, der auf sie in seinem Lehrbuch der nichteuklidischen Geometrie verweist. ${ }^{20}$

Am 04.07.1903 hielt Hausdorff seine Antrittsvorlesung in Leipzig, er war gerade zum Extraordinarius befördert worden. In Hausdorffs und auch etwas später in Liebmanns Fall war dies - salopp gesprochen - ein Titel ohne Mittel, Geld verdiente Hausdorff hauptsächlich an der Handelsakademie mit seinen dortigen Vorlesungen. Er wählte das Raumproblem zum Thema, ein Thema also, für das er sich breites Interesse erhoffen durfte. Schon in der Einleitung unterstreicht er dessen interdisziplinären Charakter. Inhaltlich geht es vor allem um die Frage, inwieweit der Raum a priori bestimmt ist. Nur für den empirischen Raum erkennt Hausdorff eine im Rahmen der Beobachtungsgenauigkeit eindeutige Bestimmtheit an, der mathematische hingegen lässt viel Spielraum; hier fallen Wendungen wie ,schöpferische

\footnotetext{
${ }^{18}$ Hausdorff war ja auch einer der ersten, wenn nicht gar der erste, der die moderne Definition von Funktion vermöge von Paaren verwandte, z. B. in seinen Grundzügen der Mengenlehre (1914, p. 33). Eine andere seinerzeit aktuelle Idee findet sich p. 483-484, wo Hausdorff in seinen Notizen versucht, eine Axiomatik der projektiven Ebene in relationentheoretischer Sprache aufzubauen - ähnlich Russell, der eine solche Grundlegung des Raumbegriffs unternahm.

19 Vgl. hierzu p. 108, wo ein entsprechendes Zitat aus „Chaos in kosmischer Auslese“ gegeben wird.

${ }^{20}$ Vgl. Liebmann [3, S. 104 n. 1]. Es geht dabei um die Kreisgeometrie in der hyperbolischen Ebene (§ 26).
} 
Freiheit unserer Gedankenbildung“ (p. 283). Mit Hilberts Axiomatik von 1899 ist nach Hausdorff klar geworden, dass es viele, gar unendlich viele, Möglichkeiten gibt, dieses System auszugestalten. Dabei ist wichtig, dass es gelungen ist, „die Abwesenheit eines Widerspruches [...] durch geeignete Abbildungen der nichteuklidischen Geometrie auf euklidische Modelle und der euklidischen Geometrie auf die reine Arithmetik direkt“ (p. 283) zu beweisen. Der Begriff „Modell“ fällt hier auf, weil er in seiner modernen Bedeutung verwendet wird. Wenn allerdings behauptet wird, „Modell“ habe in der Mathematik des 19. Jhs. immer nur für materielle Objekte gestanden (p. 144), so ist das unzutreffend. In der darstellenden Geometrie bezeichnete dieser Begriff vielmehr - modern gesprochen - das abstrakte Bild unter einer Abbildung ohne jeglichen Bezug zur Materialität. Hausdorff spricht im Zusammenhang des Konsistenzbeweises auch von „Lexikon“ (p. 155), was doch recht nah an Poincarés Wörterbuch ist - eine Metapher, die sehr erfolgreich war (und noch ist).

Ein Leipziger Trauma klingt bei Hausdorff an, wenn er schreibt (p. 293):

Daß die Dimensionenzahl drei keine Denknotwendigkeit ist, wird heute wohl gutwillig zugestanden werden; der Mathematiker wird seinerseits mit Ablehnung aller spiritistischen Zauberkunststücke zugeben, daß hier kein Erfahrungsspielraum für Abweichungen von der Drei offen bleibt.

Hier stehen die Bemühungen des Leipziger Astrophysikers K. Fr. Zöllner im Hintergrund, der um 1880 herum versuchte, spiritistische Phänomene wie das Verschwinden von Gegenständen aus geschlossenen Gefäßen oder das zerstörungsfreie Lösen von versiegelten Knoten mit Hilfe der vierten Dimension zu erklären. Dies erregte großes Aufsehen und provozierte einen Skandal, die Mathematik war genötigt, sich gegen derartigen Missbrauch zu wehren. Hierzu verwies sie auf die Freiheit ihrer Begriffsbildungen und darauf, dass diese keine Realitätsbezug für sich reklamierten. ${ }^{21}$ Pointiert formulierte Hausdorff dies so (p. 505): „Der Formalismus ist der wahre Empirismus“, womit gemeint war, dass der konsequente mathematische Formalismus der Empirie keinerlei Vorgaben macht, er greift ,der Wirklichkeit am wenigsten vor".

Als Beispiel einer nichteuklidischen Geometrie zitiert Hausdorff vor allem die sphärische, wobei die Fechner-Helmholtzschen Flächenwesen ${ }^{22}$ mehrfach anklingen (p. 288). Hier macht er eine der bei ihm eher seltenen Bemerkungen zur Geschichte (p. 288), denn es erscheint ihm merkwürdig, ,daß man die sphärische Geometrie als nichteuklidische nicht längst vor der pseudosphärischen entdeckt hat."Vielleicht war das aber gar nicht so merkwürdig. Will man die sphärische Geometrie wirklich als Alternative sehen, muss man sie ja als eigenständige Theorie, gewissermaßen als autonom, verstehen. Bis ins 19. Jh. hinein wurde sie aber als Teil der euklidischen gesehen und konsequenterweise auch als Sphärik und eben nicht als sphärische

\footnotetext{
${ }^{21}$ Vgl. Volkert [7, S. 133-182]. Auf p. 385 spricht Hausdorff nochmals den Spiritismus an im Kontext höherer Dimensionen und Mannigfaltigkeiten.

22 Diese werden auch bei O. Liebmann erwähnt; vgl. Zitat p. 43. Zu diesen und ähnlichen Ideen vgl. Volkert ([7], insbesondere 31-32). Gelegentlich wird in diesem Kontext auch Th. Reid genannt, vgl. hierzu die Diskussion im einleitenden Essay pp. 13-14.
} 
Geometrie bezeichnet. ${ }^{23}$ Zudem widersprach sie offensichtlich dem fundamentalen Axiom „Zwei Geraden schließen keinen Flächenraum ein“, wich also nicht nur in der Parallelenfrage von der euklidischen Geometrie ab. Angesichts Hausdorffscher Präzision erstaunt es, dass er hier nicht genauer auf die elliptische Geometrie, wie er das z. B. in seinem unveröffentlichten Aufsatz zur nichteuklidischen Geometrie tut, ausführlicher eingeht; vielleicht war dies der Rücksichtnahme auf sein Publikum geschuldet, das ja nicht nur aus Mathematikern bestand. ${ }^{24}$

Insgesamt ist Hausdorffs Antrittsvorlesung ein Plädoyer für die Autonomie der Mathematik, insbesondere der Geometrie, als Theorie, nur in ihren Anwendungen tritt ein empirisches Element hinzu:

Als Komplex freigewählter Axiome mit denknotwendigen Konsequenzen, als „hypothetisch-deduktives System“ ist die Geometrie eine rein logische Konstruktion; darin aber, daß ein bestimmtes Axiomensystem gegenüber anderen gleichberechtigten zu Folgerungen führt, die sich zur Beschreibung realer Vorgänge als geeignet erweisen, darin liegt natürlich ein empirisches Element. (p. 299-300)

Die Unterscheidung von abstrakter Geometrie und angewandter, also interpretierter, Geometrie hatte auch Poincaré schon vielfach hervorgehoben. ${ }^{25}$ Wenn wir so reden, als gehorchten Gegenstände unserer Erfahrung den Gesetzen der Geometrie, wenn wir also die eben markierte Unterscheidung nicht vornehmen, so müssen wir uns darüber im Klaren sein, dass dies eine Rede ,als ob“ ist (p. 299) - eine hübsche Anspielung Hausdorffs auf Vaihingers seinerzeit populäre „Philosophie des Als-ob“ und ihre nützlichen Fiktionen (wie etwa Atome).

Hausdorffs Antrittsvorlesung wurde nicht, wie meist üblich, im Jahresbericht der DMV abgedruckt sondern in einer philosophischen Zeitschrift, nämlich in Ostwalds Annalen der Naturphilosophie. Spuren von Hausdorffs Antrittsvorlesung findet man wieder bei H. Liebmann. Er zitiert sie in seinem Lehrbuch der nichteuklidischen Geometrie mit der Behauptung ihres Autors ,,daß die Vorstellbarkeit der nichteuklidischen Geometrie unter geeigneten persönlichen Vorbedingungen entschieden zu bejahen ist. "26 Hausdorff wird auch in Liebmanns Antrittsvorlesung mit dem Raumproblem zitiert, wieder im Kontext der Veranschaulichungsfrage: Die Vorstellbarkeit der nichteuklidischen Geometrie ist eine Frage der Gewöhnung (also ein Problem der Psychologie könnte man hinzufügen).

\footnotetext{
23 Bei Chr. Gudermann finden sich in seinem Lehrbuch der niederen Sphärik (1836) deutliche Ansätze in die oben bezeichnete Richtung einer eigenständigen sphärischen Geometrie.

24 Etwas genauer wird er dann auf p. 292, wo er auf die Einseitigkeit der projektiven Ebene und die bekannten inkongruenten Gegenstücke Kants eingeht; im Entwurf eines Artikels über die nichteuklidische Geometrie (p. 349-389) wird die elliptische Geometrie ausführlich behandelt bis hin zu den Cliffordschen Parallelen, ihrer Abgrenzung gegen die sphärische Geometrie und Studys parataktischen Geraden (p. 375); auch in den Notizen wird sie öfter diskutiert (vgl. etwa p. 492).

25 Vgl. auch p. 355, wo Hausdorff sogar wörtlich von ,zuordnen“ spricht; später wird dieser Punkt im logischen Empirismus, im einleitenden Essay wird vor allem M. Schlick erwähnt, zum Problem der Zuordnungsdefinitionen führen. Weitere Anklänge an Poincaré findet man p. 298, wo die Starrheit eines Körpers als zweckmäßige Annahme geschildert wird.

26 Liebmann [3, S. 16 n. 1]. Weiter heißt es: „Er [Hausdorff; K. V.] beruft sich dabei auf Helmholtz.“.
} 
Unter den publizierten Texten von Hausdorff, die im vorliegenden Band wieder abgedruckt werden, finden sich mehrere Besprechungen, z. B. die bereits erwähnte des Buches von W. Ostwald. Ausführlich und mathematisch anspruchsvoll ist die von E. Studys „Geometrie der Dynamen“, die zeigt, dass Hausdorff tief in Materien wie Liniengeometrie sowie projektive und nichteuklidische Geometrie eingedrungen und mit deren technischen Details vertraut war. Study und Hausdorff wurden später Kollegen in Bonn, eine Gemeinsamkeit der beiden war wohl ihre Abneigung gegen den ,großen Klein“. ${ }^{27}$ Ihm, seinem „Freund“, hat Hausdorff einen Nachruf (1930) gewidmet, der ebenfalls im vorliegenden Band abgedruckt ist. Hatte P. Mongré seit 1913 geschwiegen, so kam er bei dieser Gelegenheit doch wieder zu Worte, denn hier spricht Zarathustra und die südlichen Landschaften werden beschworen. Ein ausgeprägtes Pathos war Hausdorff nicht fremd.

Auffallend bei Hausdorffs Rezensionen ist, dass er vor obskuren Texten nicht zurückschreckte, etwa J. B. Stallo's „Die Begriffe und Theorien der modernen Physik“. Vorurteilsfrei aber doch kritisch versuchte er, auch solchen Publikationen gerecht zu werden. Das Spektrum der von Hausdorff besprochenen Bücher ist breit, es reicht von der Willensfreiheit (R. Manno) bis zur Natur der Dinge an sich (W. K. Clifford) und der Logik (M. Palági).

Schließlich folgen unpublizierte Texte aus Hausdorffs Nachlass. Von besonderem Interesse ist hier sein Entwurf eines Artikels über nichteuklidische Geometrie ,für eine allgemeine Naturforscherzeitung“ (p. 349) - also nicht für Fachleute. Hier zeigt der Verfasser großes Geschick, wenn es darum geht, schwierige Sachverhalte allgemein verständlich darzustellen. Dieser lange Text greift mehrere Punkte wieder auf, die in dieser Besprechung schon erwähnt wurden, z. B. die empirische Gültigkeit der euklidischen Geometrie (p. 350) und die Verteidigung der Mathematik gegen philosophische Übergriffe (p. 351) - gipfelnd im Zitat von Georg Cantor „Das Wesen der Mathematik liegt in ihrer Freiheit“" (p. 351).

Eine stichwortartige Ausarbeitung für die von Hausdorff im WS 1903/04 in Leipzig ,,auch für Nicht-Mathematiker“28 gehaltene Vorlesung über ,Zeit und Raum“, die nicht-technisch, wohl aber recht philosophisch gewesen zu sein scheint, eine Studie zum Relativitätsprinzip und Fragmente aus dem Nachlass zu geometrischen Fragen runden diesen reichhaltigen Band ab. Diese formulieren stichwortartig interessante Ideen, etwa zum „Transformationsprinzip“ (p. 506), verbunden mit dem Streben nach größter Allgemeinheit (konkret: Verzicht auf Stetigkeit und Eindeutigkeit).

Insgesamt ist Band VI der Hausdorffschen Werke eine sehr lohnende Quelle für alle, die sich für Hausdorff und/oder für die geistige Situation kurz nach der Jahrhundertwende interessieren. Sowohl den Herausgebern der Werke als auch dem Bearbeiter des Bandes ist dafür zu danken, dass diese nun zugänglich ist.

\footnotetext{
${ }^{27}$ Zu Study vgl. man die Dissertation von Yvonne Hartwich „Eduard Study (1862-1930) - ein mathematischer Mephistopheles im geometrischen Gärtchen“ (Johannes Gutenberg Universität Mainz 2006) [https:// openscience.ub.uni-mainz.de/handle/20.500.12030/3484]. Hausdorffs Besprechung von Study wird übrigens von Liebmann in seiner Antrittsvorlesung zitiert mit ihren Ausführungen zur Wichtigkeit von geeigneten Zeichensystemen.

$28 \mathrm{Vgl}$. https://histvv.uni-leipzig.de/dozenten/hausdorff_f.html - eine sehr nützliche Auflistung aller von Hausdorff in Leipzig gehaltenen Vorlesungen. Diese Ausarbeitung wird in Epples Einleitung (pp. 142-156) behandelt.
} 
Note added in proofs: Bzgl. Leipziger Antrittsvorlesungen vgl. man „Leipziger mathematische Antrittsvorlesungen. Auswahl aus den Jahren 1869 - 1922“, hg. von H. Beckert und W. Purkert (Leipzig: Teubner, 1987).

Funding Open Access funding enabled and organized by Projekt DEAL.

Open Access Dieser Artikel wird unter der Creative Commons Namensnennung 4.0 International Lizenz veröffentlicht, welche die Nutzung, Vervielfältigung, Bearbeitung, Verbreitung und Wiedergabe in jeglichem Medium und Format erlaubt, sofern Sie den/die ursprünglichen Autor(en) und die Quelle ordnungsgemäß nennen, einen Link zur Creative Commons Lizenz beifügen und angeben, ob Änderungen vorgenommen wurden.

Die in diesem Artikel enthaltenen Bilder und sonstiges Drittmaterial unterliegen ebenfalls der genannten Creative Commons Lizenz, sofern sich aus der Abbildungslegende nichts anderes ergibt. Sofern das betreffende Material nicht unter der genannten Creative Commons Lizenz steht und die betreffende Handlung nicht nach gesetzlichen Vorschriften erlaubt ist, ist für die oben aufgeführten Weiterverwendungen des Materials die Einwilligung des jeweiligen Rechteinhabers einzuholen.

Weitere Details zur Lizenz entnehmen Sie bitte der Lizenzinformation auf http://creativecommons.org/ licenses/by/4.0/deed.de.

\section{Literatur}

1. Hölder, O.: Anschauung und Denken in der Geometrie. Akademische Antrittsrede gehalten am 22. Juli 1899. Teubner, Leipzig (1900). Nachdruck Darmstadt: WB, 1968

2. Hilbert, D.: Grundlagen der Geometrie. Springer, Berlin-Heidelberg (2015). hg. von K. Volkert

3. Liebmann, H.: In: Notwendigkeit und Freiheit in der Mathematik. Akademische Antrittsrede gehalten in Leipzig am 25. Februar 1905 Jahresbericht der Deutschen Mathematiker-Vereinigung, Bd. 14, S. 230-248. (1905)

4. Lorey, W.: Das Studium der Mathematik an deutschen Universitäten seit Anfang des 19. Jahrhunderts. Teubner, Leipzig und Berlin (1916)

5. Voelke, J.-D.: Renaissance de la géométrie non euclidienne entre 1860 et 1920. Peter Lang, Bern und Frankfurt a. M. (2005)

6. Volkert, K.: Das Undenkbare denken. Die Rezeption der nichteuklidischen Geometrie im deutschsprachigen Raum (1860-1900). Springer, Berlin-Heidelberg (2010)

7. Volkert, K.: In höheren Räumen. Der Weg der Geometrie in die vierte Dimension. Springer, Berlin (2018) 